\title{
Sub-picosecond microjoule-class fiber lasers
}

\author{
C. Lecaplain ${ }^{1}$, B. Ortaç ${ }^{2}$, G. Machinet ${ }^{3}$, J. Boullet ${ }^{3}$, M. Baumgartl ${ }^{4}$, T. Schreiber ${ }^{5}$, E. Cormier ${ }^{3}$, A. Hideur ${ }^{1}$, \\ ${ }^{1}$ CORIA UMR 6614, Université de Rouen, Avenue de l'université BP 12, 76801 Saint Etienne du Rouvray, France \\ ${ }^{2}$ UNAM-Institute of Materials Science and Nanotechnology, Bilkent University, 06800 Bilkent, Ankara, Turkey \\ ${ }^{3}$ Université de Bordeaux-CNRS-CEA, CELIA, 351 cours de la Libération F-33405 Talence, France \\ ${ }^{4}$ Institute of Applied Physics, Friedrich Schiller University, Albert-Einstein-Strasse 15, D-07745 Jena, Germany \\ ${ }^{5}$ Fraunhofer Institute for Applied Optics and Precision Engineering, Albert-Einstein-Str. 7, D-07745 Jena, Germany \\ Tel.: +33232953 736, E-mail: lecaplain@ coria.fr
}

\begin{abstract}
We study the impact of the mode-locking mechanism on the performances of a microjoule-class all-normal dispersion fiber laser featuring large-mode-area photonic crystal fibers. (C)2011 Optical Society of America

OCIS codes: (140.3510) Lasers, fiber, (320.7090) Ultrafast lasers, (060.5530) Pulse propagation and solitons.
\end{abstract}

\section{Introduction}

In recent years, femtosecond fiber lasers have been developed with ever increasing performances in terms of pulse duration and energy. Thanks to the advances in understanding of new pulse shaping mechanism, as well as in development of low-nonlinearity large-mode-area fiber technology, fiber lasers are now emerging as competitive ultrashort sources. The fundamental challenge for ultrafast fiber lasers relies on the control of the excessive nonlinearity which hinders a self-consistent pulse evolution at high-energy levels. An increase of pulse energy from fiber oscillators can be achieved by stretching the pulse during its propagation while reducing the accumulated nonlinear phase. This concept is pursued in operation regimes such as stretched-pulse, self-similar or all-normal dispersion lasers [1-3]. The mode area size determines the amount of accumulated nonlinear phase. Consequently, the employment of low-nonlinearity large-mode-area photonic crystal fibers (PCF) enables significant power scaling. Fiber oscillators that reach the megawatt peak powers have been reported based on this approach [4-5]. Notably, the extension of this approach to photonic crystal rods opens the road for sub-picosecond microjoule-class fiber sources [6-7]. In this communication, we report the generation of high-energy sub-picosecond pulses from a highly normal dispersion fiber laser featuring an Yb-doped rod-type PCF and a large-mode-area PCF. We discuss the different regimes of operation obtained in these new class of fiber oscillators which depend principally on the the force of the mode-locking mechanism. Output average powers as high as $15 \mathrm{~W}$ at $15 \mathrm{MHz}$ repetition rate, corresponding to $1 \mu \mathrm{J}$ energy are achieved.

\section{Experiment and results}

The typical experimental setup of the passively mode-locked fiber oscillator is shown in Fig.1. The gain fiber consists in a $1 \mathrm{~m}$ long Yb-doped photonic crystal fiber with a single-mode core diameter of more than $80 \mu \mathrm{m}$. The laser cavity is mounted in sigma-configuration around a polarization-sensitive isolator. The large-mode-area photonic crystal fiber is cladding-pumped with a fiber-coupled laser diode emitting $50 \mathrm{~W}$ at $976 \mathrm{~nm}$. A 7 m long passive LMA microstructure fiber with a core diameter of $25 \mu \mathrm{m}$ is inserted inside the cavity, leading to a total cavity dispersion of about $0.152 \mathrm{ps}^{2}$. Insertion of the passive fiber after the output coupling allows controlling both the dispersion and the accumulated nonlinear phase along the cavity. Passive mode locking is achieved using fast saturable absorber mirrors (SAM). Different SAM structures featuring high modulation depths of $>20 \%$, saturation fluences of $\sim 100$ $\mu \mathrm{J} / \mathrm{cm}^{2}$ and fast relaxation time $<500 \mathrm{fs}$ are studied. The anti-resonant SAM structures ensures an absorption bandwidth of more than $45 \mathrm{~nm}$ while the resonant structures exhibit $\sim 20 \mathrm{~nm}$ bandwidth. In addition, several waveplates are introduced behind and after the gain fiber to control the beam polarization. This allows to optimize the parameters of the nonlinear polarization evolution (NPE) process which could contribute to pulse shaping. The laser produces a stable self-starting pulse train at a repetition rate of $15 \mathrm{MHz}$.

The results obtained with the anti-resonant SAM structure for an average output power of $13 \mathrm{~W}$ are shown on Fig. 2. The laser delivers highly-chirped picosecond pulses with $\sim 10 \mathrm{~nm}$ spectral widths. The output pulses are extra-cavity dechirped to $550 \mathrm{fs}$ duration using transmission gratings [Fig. 2]. This duration is 1.7 times higher than the transformlimited duration calculated from the spectrum (327 fs). The corresponding peak power is higher than 1.2 MW. The radiofrequency measurements reveal a good amplitude stability of the pulse train. The pulse to pulse energy fluctuations are estimated to be less that $0.4 \%$. We note that the current laser performances are limited to $\sim 1 \mu \mathrm{J}$ by 


\section{CThM1.pdf}

the available pump power.

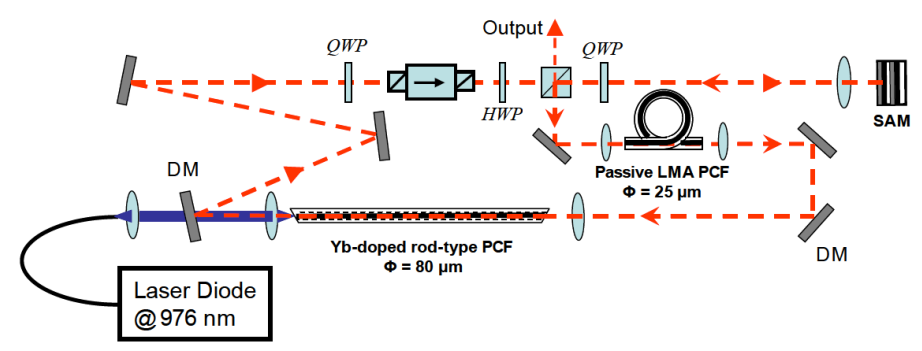

Fig. 1: Experimental setup of the chirped-pulse fiber oscillator

By increasing the force of the mode-locking mechanism through the combination of the SAM with the NPE process, we obtain shorter pulses with slightly boarder spectra. Indeed, the output pulse duration is decreased from $30 \mathrm{ps}$ to less that 13 ps by adjusting the wave-plates settings. The dechirped pulse duration is then shortened to $450 \mathrm{fs}$.
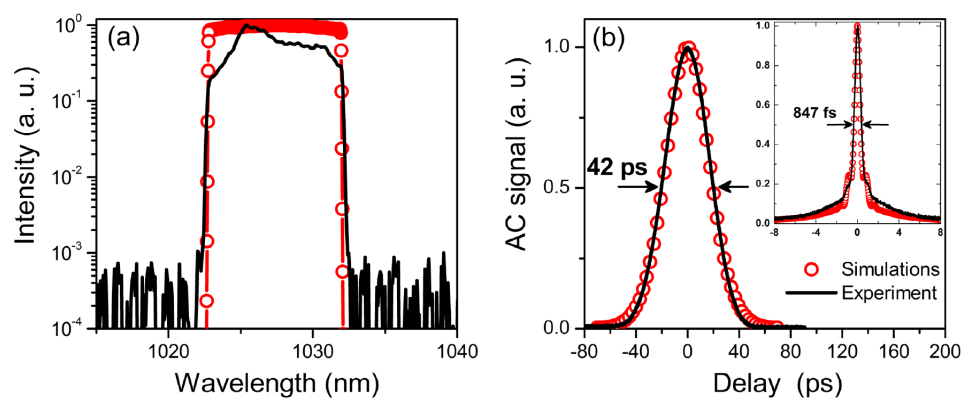

Fig. 2 : Output of the laser : typical output spectrum (a) and (b) output pulse autocorrelation. Inset : dechirped pulse autocorrelation.

In a second experiment, we use a resonant SAM structure with $20 \mathrm{~nm}$ bandwidth. In this case, the laser generates 7 ps pulses with about $11 \mathrm{~W}$ of average power. The spectral width is then about $12 \mathrm{~nm}$. The output pulses are compressed down to $300 \mathrm{fs}$. This result reveals that the spectral filtering induced by the limited bandwidth of the SAM is interesting for pulse shortening. However, this introduced an additional limit in terms of pulse energy. To study the laser capabilities for energy scaling, numerical simulations were performed considering the laser set-up shown on Fig. 1. Numerical simulations are in good agreement with experiments and predict that stable pulse solutions do exist for more than $10 \mu \mathrm{J}$ intra-cavity pulse energy. More discussions on the impact of the mode-locking mechanism on the laser performances will be addressed.

\section{Acknowledgement}

This work is supported by the Inter Carnot-Fraunhofer program under project APUS and the French Agency for Research under project OFFEMET. We acknowledge support from the Conseil Régional de Haute Normandie.

\section{References}

[1] K. Tamura, E. P. Ippen, H. A. Haus, and L. E. Nelson, "77-fs pulse generation from a stretched-pulse mode-locked all-fiber ring laser," Opt. Lett. 18, 1080-1082 (1993).

[2] F. Ö. Ilday, J. R. Buckley, W. G. Clark, and F. W. Wise, "Self-Similar Evolution of Parabolic Pulses in a Laser," Phys. Rev. Lett. 92, 213902 (2004).

[3] L. M. Zhao, D. Y. Tang, and J. Wu, "Gain-guided soliton in a positive group-dispersion fiber laser," Opt. Lett. 31, 1788-1790 (2006).

[4] S. Lefrançois, K. Kieu, J. Deng, J. D. Kafka and F. W. Wise "Scaling of dissipative soliton fiber lasers to megawatt peak powers by use of large-area photonic crystal fiber", Opt. Lett. 35, 1569-1571 (2010).

[5] M. Baumgartl, B. Ortaç, C. Lecaplain, A. Hideur, J. Limpert and A. Tünnermann, "Sub-80 fs dissipative soliton large mode area fiber laser", Opt. Lett. 35, 2311-2313 (2010).

[6] B. Ortaç, M. Baumgartl, J. Limpert, and A. Tünnermann, "Approaching microjoule level pulse energy with mode-locked femtosecond fiber lasers," Opt. Lett. 34, 1585-1587 (2009).

[7] C. Lecaplain, B. Ortaç, G. Machinet, J. Boullet, M. Baumgartl, T. Schreiber, E. Cormier, and A. Hideur, "High-energy femtosecond photonic crystal fiber laser," Opt. Lett. 35, 3156-3158 (2010). 